\title{
APPLICATIONS OF NARROW-BAND PHOTOMETRY IN THE STUDY OF PECULIAR RED GIANTS
}

\author{
ROBERT F. WING \\ Ohio State University, Columbus, OH 43210, U.S.A.
}

\begin{abstract}
Narrow-band photometry, carried out with filters or spectrum scanners, is useful for measuring molecular bandstrengths and continuum energy distributions in late-type stars. This review emphasizes observations by the writer on three different multicolor photometric systems in the near infrared $(0.75-4.0 \mu \mathrm{m})$; a summary of available data is given. While applications to date have been primarily qualitative (classification, recognition of peculiarities, relative temperatures), future applications are expected to be quantitative (determinations of effective temperatures, luminosities, and abundances) and based upon comparison with synthetic spectra.
\end{abstract}

\section{Introduction}

Narrow-band photometry is a technique that provides both photometric information (magnitudes and colors) and spectroscopic data (the strengths of spectral features) in a single observation. This simultaneity of measurement of colors and feature strengths is an important advantage in work on variable stars, as well as a great convenience. The technique is especially well suited to studies of cool stars since spectral regions that are heavily depressed by molecular band absorption - and the relatively clear intervals between the bands - are often on the order of 50-100 $\AA$ wide and can be measured conveniently by a number of methods. Applications to latetype stars thus include both measurements of molecular band strengths and measurements of continuum colors.

In fact, applications of narrow-band photometry have been somewhat miscellaneous, often serving as supplemental to other data. I will not attempt a complete listing of relevant observations; rather, I will use this opportunity to discuss the special advantages of the technique, to give examples of interesting applications, to summarize the extensive but largely 
unpublished sets of data that are now available on certain well-defined systems of narrow-band photometry, and to suggest and encourage applications of the available data. Because of major advances in atmospheric models and line lists relevant to late-type stars (see, for example, Plez 2000), many of the older data sets are now finding important new applications.

In the sections that follow, I discuss data obtained by the writer and his colleagues with three kinds of equipment: (1) scanner observations with an S-1 photomultiplier at selected wavelengths in the near infrared out to $1.1 \mu \mathrm{m}$; (2) filter photometry in the same spectral region; and (3) scanner observations with an InSb detector extending the wavelength range longward to about $4 \mu \mathrm{m}$. All three are single-channel instruments, with which the different wavelengths must be measured sequentially. To a large extent these instruments are no longer supported and have been replaced by spectrographs with CCDs and infrared array detectors. When I started these programs, there was no alternative to the single-channel instruments, and frankly I was not concerned by their "inefficiency" since many thousands of stars are so bright that good photometric data could be acquired at a large number of wavelengths in reasonable amounts of time on a small telescope. That, of course, is still true, and since so much work on bright stars remains to be done, it is unfortunate that it is becoming difficult or impossible to continue observing in the same manner, thereby staying on precisely the same photometric systems.

Many of my remarks, however, apply equally well to spectra obtained with either single-channel or array detectors. The difference is basically in what you take home with you from the telescope. With a single-channel instrument, you must decide in advance which wavelengths are to be measured, and the time at the telescope is spent integrating at those wavelengths only. With an array detector, you record the whole spectrum (over some wide range) at the telescope, and do the photometry at home on the computer. The end result is often the same. By recording the complete spectrum, you can analyze it in different ways and change your mind as often as you please about which wavelengths to use in the analysis, but at the cost of dealing with much larger data sets. For bright-star work, I still prefer the single-channel approach, not only because the data sets are so much smaller but also because it helps to ensure that the results will be expressed on a stable, well-defined photometric system.

Well, it is not my purpose here to discuss the relative merits of different observing techniques, and it would be pointless to advocate observing by methods that are no longer supported at the major observatories. Rather, my purpose is to describe data sets that already exist but have not yet been fully exploited. Most of the applications that I've pursued to date have involved extracting information directly from the data, without input from 
other sources. Examples include spectral classifications based on molecular band strengths, comparisons of the continuum slopes (i.e. temperatures) of $\mathrm{M}, \mathrm{S}$, and $\mathrm{C}$ stars, discussions of chemical composition differences between stars and recognition of peculiarities based on bandstrength-color diagrams, and determinations of the reddening and distances of $\mathrm{M}$ supergiants. On the other hand, quantitative interpretations of the photometry had to wait for the appearance of model atmospheres and line lists capable of reproducing the spectra of cool stars. Applications based on comparisons of observed and synthetic photometry - including, for example, determinations of effective temperatures and certain abundance ratios - are only beginning, and some specific suggestions along these lines are outlined in the final section.

\section{Scanner Observations in the $1 \mu \mathrm{m}$ Region}

My long-term interest in studying late-type stars by means of narrow-band photometry in the near infrared began abruptly in July 1965, when an automated single-channel spectrum scanner designed by E. J. Wampler became available for use at Lick Observatory's Crossley telescope. The timing was perfect for me, because I was a Berkeley student looking for a thesis topic. I had been studying the blue/visual spectra of very cool stars (especially Miras) on the basis of high-dispersion spectra and various kinds of photometry but had become discouraged by the difficulty of extracting useful information from the jumble of atomic and molecular features that occur at short wavelengths. Wampler's scanner, however, when used with an S-1 photocell, gave access to the far-red/near-infrared region out to $1.1 \mu \mathrm{m}$. This region is nearly devoid of strong atomic lines but contains strong bands of a number of different molecules, usually with stretches of relatively clean continuum between them. A decision was quickly made to explore this region, measuring molecular band strengths and continuum slopes in as many kinds of late-type stars as possible (Wing 1967).

The observed colors of cool stars on almost any photometric system are strongly affected by molecular absorption, which is due to almost completely different sets of molecules in stars of types M, S, and C. Thus one of the major observational problems in studying late-type stars is to find color indices that can serve as reliable temperature indicators, unaffected by composition differences.

Using Wampler's scanner first in the continuous-scan mode (with integrations every $10 \AA$ through a $20 \AA$ exit slot), I explored the spectra of representative cool stars from 0.75 to $1.10 \mu \mathrm{m}$. It was then possible to select the best continuum points for each type of spectrum, as well as the points most sensitive to the presence of various molecules. Unfortunately, 
the most useful wavelength points in stars of types $\mathrm{M}, \mathrm{S}$, and $\mathrm{C}$ have very little overlap. Even among the normal $M$ stars, one would chose different continuum points in early $M$ and late $M$ stars. Also, for the purpose of measuring molecular band strengths, it is desirable to include both the intrinsically strongest bands of each molecule (to be able to measure them when weak) and also some weaker bands (to avoid saturation when the absorption is stronger). Thus a general program intended to be useful for all kinds of late-type stars necessarily involves a large number of wavelengths. The scans given in my thesis (Wing 1967) consist of integrations first at 26 , and later at 27 , wavelengths. The bandpass used throughout was $30 \AA$.

The observations were reduced as 27-color all-sky photometry, with extinction and transformation coefficients determined in the usual manner. The data were transformed to a system of absolute fluxes by assuming a model-atmosphere energy distribution for $\alpha$ Lyr.

When the calibrated flux measurements are plotted against wavelength, the upper envelopes of points are seen to follow blackbody curves quite closely in most kinds of cool stars (exceptions being the very late $\mathrm{M}$ and $\mathrm{S}$ stars, later than about M7). That is, there are enough data points falling close enough to the same blackbody curve that one can believe that this blackbody curve is a reasonable approximation to the star's actual line-free continuum, and that the temperature of this blackbody is related to the effective temperature of the star. However, this blackbody continuum is defined by different points in different stars: no simple color index (magnitude difference) can be defined from the 27-color data that would provide a blanketing-free temperature index in stars of all kinds. I therefore developed a numerical scheme for fitting blackbody curves to the scans as part of the standard reduction procedure. The continua so obtained provide both a color temperature and a reference continuum with respect to which the strengths of absorption features could be measured. An important advantage of measuring absorption strengths vertically, with respect to the local continuum, is that such indices are reddening-free, so that spectral classifications and other quantities derived from them are also reddening-free. The color temperatures from blackbody fits are, of course, affected by reddening, but they are obtained in a consistent manner in stars of all compositions.

In practice, I have used the "inverse color temperature" $\theta_{\mathrm{c}} \equiv 5040 / T_{\mathrm{c}}$ as my continuum color index. This quantity is proportional to conventional color indices (magnitude differences) and hence can be used in the same way.

Approximately 1300 scans on the 27-color system were obtained over a period of 2.5 years. Program stars included examples of essentially all kinds of stars cooler than the Sun - i.e. all spectral types, luminosity classes, variability types, and chemical peculiarities. The tables in Wing 

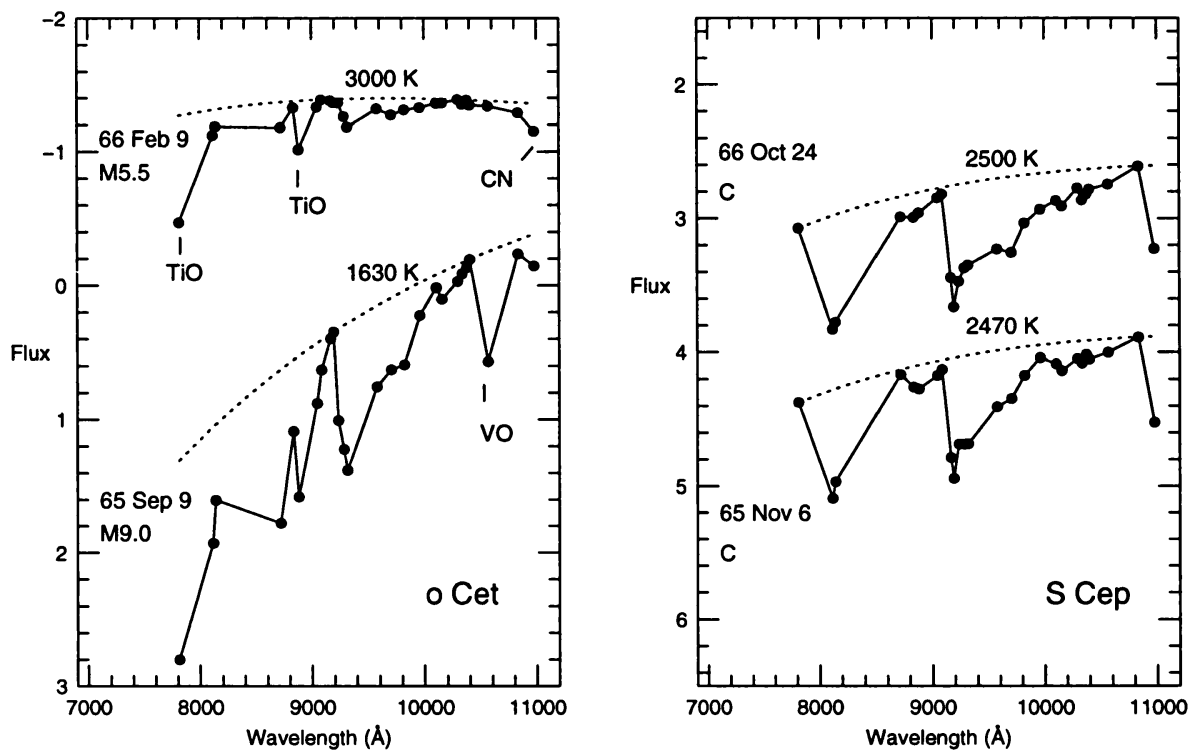

Figure 1. Scanner observations of Mira variables. The M-type variable $o$ Cet (left) and the C-type variable S Cep (right) are each shown near the times of maximum and minimum light. Measurements taken through a $30 \AA$ bandpass are given on an absolute flux scale and fitted with blackbody curves. Molecules responsible for absorptions in the $\mathrm{M}$ star are labeled; nearly all the absorption in the $\mathrm{C}$ star is due to $\mathrm{CN}$.

(1967) give averaged data for 155 non-variable stars observed an average of $\sim 3$ times each and 808 individual observations of 160 variable stars. In particular, several Mira variables were observed 20 or more times over 2 or 3 cycles, and these observations showed that Miras execute enormous loops - both clockwise and counter-clockwise - in diagrams of bandstrength vs. continuum color (Spinrad \& Wing 1969; Wing \& Yuan 1998).

Four examples of scanner "spectra" are shown in Figure 1. At the left are scans of $o$ Cet obtained at maximum and minimum light, when the observed spectral types were M5.5 and M9.0, respectively. They illustrate the well-known fact that as an M-type Mira gets fainter, it also gets cooler, as indicated not only by the growth of the $\mathrm{TiO}$ and $\mathrm{VO}$ bands but also by the considerable change in continuum slope. At the right is shown a similar pair of scans of S Cep, a C-type Mira, also observed close to the times of maximum and minimum visual light. In this case nearly all the absortion is due to the red system of CN. Note that the maximum-light and minimum-light spectra are nearly identical. It is well known that $\mathrm{CN}$ is not very sensitive to temperature and that the spectra of $\mathrm{C}$-type Miras change relatively little with phase; but what is surprising is that the observed continuum slope hardly changed either, suggesting that the temperature 
itself remained constant.

It is hard to imagine how a Mira can change in infrared brightness by a factor of two or more without an accompanying change in temperature. It seems to me that the calibrated scans of Fig. 1 are telling us that we have a problem in the measurement of color temperatures for either M-type or C-type stars (or both), and consequently that the color temperatures obtained for $\mathrm{M}$ and $\mathrm{C}$ stars by blackbody continuum fitting do not successfully place them on the same scale. Possibly the residual $\mathrm{CN}$ absorption in the continuum points varies with temperature in just such a way as to cancel the effect of a changing blackbody continuum slope. This question can, I hope, be settled by synthetic spectrum calculations.

The reason for discussing these old observations here is that they are, I believe, still useful. Or, I should say, they have recently become useful again, after a long lapse. During the 1970s all 75 nights of scanner observations were re-reduced with the help of improved standard fluxes and a new absolute flux calibration. Then, with the changes in technology that occurred in the late 1970s, the punched cards containing these data became unreadable, and for 15 years I did nothing further with them, concentrating instead on the filter photometry described in the next section. Recently, however, my university's computer center briefly acquired a refurbished card-reader so that people like me could have "one last chance" to transfer their data to magnetic media. At the same time I became aware of the great advances being made in the atmospheric modeling of cool stars, making possible many interesting applications of the data that had not been feasible before. I therefore went to considerable pains to coax several tens of thousands of 30-year-old punched cards through the card reader, and I am happy to report that my scanner observations have been fully restored. All scans reported in Wing (1967), as well as those acquired subsequently, are now accessible at the terminal - no more hauling dollyfuls of cards to the computer center across campus - and can be sent to interested colleagues by e-mail. Now at last it should be possible to carry out some of the objectives for which these observations were originally intended - in particular, that of placing the color temperatures of $\mathrm{M}$ and $\mathrm{C}$ stars on a single scale that is independent of molecular band strength.

\section{Eight-Color Photometry}

Since 1969, without ready access to a spectrum scanner, I have used narrowband filter photometry to pursue similar objectives. My eight-color photometric system (Wing 1971), which uses interference filters of $\sim 50 \AA$ bandwidth, is largely based on the 27-color scanner program and is intended to measure continuum colors and bandstrengths of $\mathrm{TiO}, \mathrm{VO}$, and $\mathrm{CN}$ in 

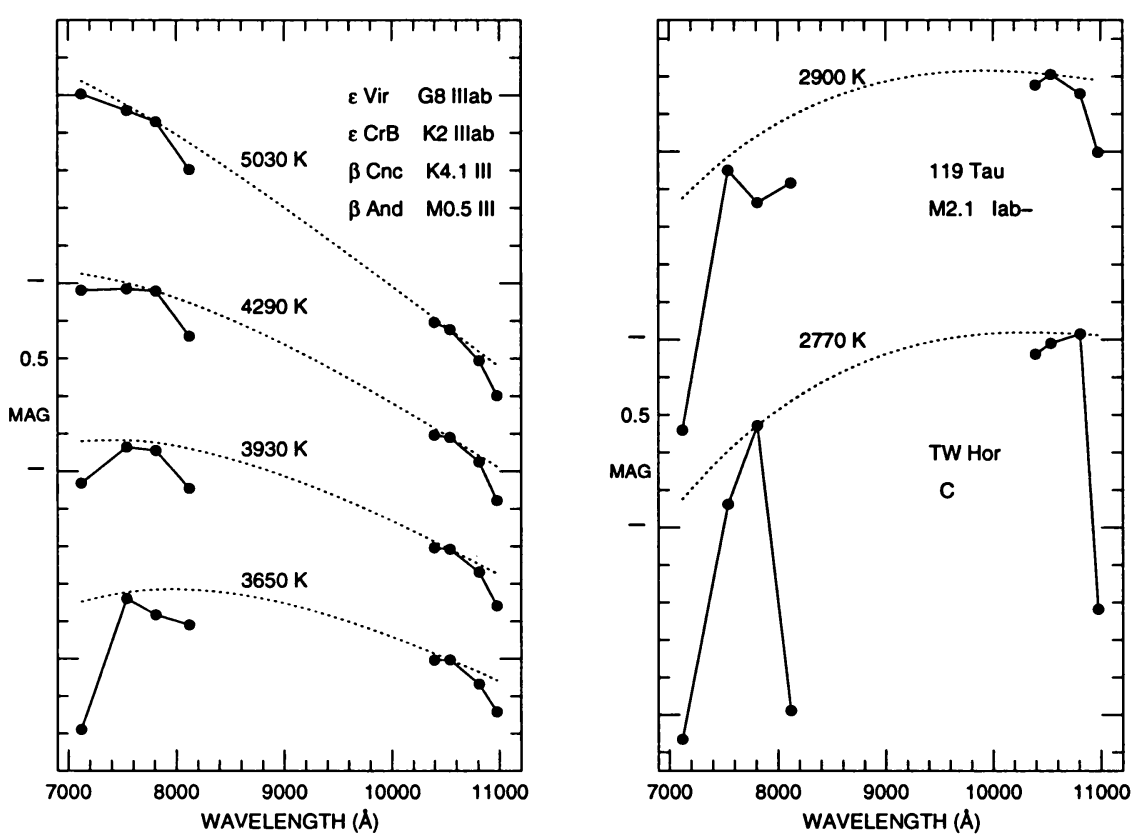

Figure 2. Eight-color observations of giants of types $\mathrm{G}, \mathrm{K}$, and $\mathrm{M}$, an $\mathrm{M}$ supergiant, and a carbon star. The C-type spectrum is essentially a map of $\mathrm{CN}$ absorption; TiO, when present, depresses filters 1 and 3. Data are reduced to an absolute flux system and plotted with arbitrary vertical shifts. Each spectrum is compared to a blackbody curve of the indicated temperature.

the $0.71-1.10 \mu \mathrm{m}$ spectra of $\mathrm{M}$ and $\mathrm{C}$ stars. In reducing the number of wavelengths from 27 to 8 , it did not seem possible to do a good job of studying the more varied spectra of stars of type $S$; the idea was to include the best continuum points and the strongest molecular bands in both $\mathrm{M}$ and $\mathrm{C}$ stars, and this can indeed be done with 8 filters in all but the most extreme cases. The collection and use of data on this system has been my main observational activity for nearly 30 years. I don't have an up-to-date count, but an estimate made several years ago indicated that approximately 30000 observations of about 15000 different stars had been made on this system - mostly by the writer, but with significant contributions from 8 or 9 collaborators, most notably N. M. White, J. H. Baumert, S. B. Yorka, and E. Costa. Details of the photometric system and its associated calibrations are given in MacConnell, Wing \& Costa (1992).

The information content of the eight-color photometry is best described with the help of some plotted spectra (Figure 2). It is not customary for photometrists to refer to their data as "spectra," but since this photometry is expressed on an absolutely calibrated system, a plot of the observed 
magnitudes vs. wavelength is indeed a simple spectrum, and spectroscopic (reddening-free) quantities can be derived by measuring the depression of any point, in magnitudes, with respect to the continuum at that wavelength.

In $\mathrm{G}$ and $\mathrm{K}$ stars, through type $\mathrm{K} 3$, the only molecule affecting the eightcolor spectrum is CN. This is also nearly true of most carbon stars, so that, for example, the K2 and C stars in Fig. 2 have the same characteristic shape apart from overall differences in band strength and temperature. The K4 and M0 giants and the M2 supergiant, however, are affected by $\mathrm{TiO}$ as well as $\mathrm{CN}$; in these cases the continuum does not pass through any of the first four filters because it has been set by an iterative procedure which allows for residual $\mathrm{CN}$ absorption in filter 2 to obtain a better continuum point than the TiO-contaminated filter 3 .

For normal M stars of all luminosities, the eight-color system is wellsuited to providing high-quality, two-dimensional spectral classifications, due to the fortunate circumstance that the two strongest molecular band systems in the $1 \mu \mathrm{m}$ region, those of $\mathrm{TiO}$ and $\mathrm{CN}$, are sensitive to temperature and luminosity, respectively. Classifications on the eight-color system are considerably improved over what could be obtained from the 27-color scans because the filter system extends to a shorter wavelength to include the strong $\gamma(0,0)$ band of $\mathrm{TiO}$ near $0.71 \mu \mathrm{m}$. Wing and Yorka (1979) have shown that the eight-color classifications of $\mathrm{M}$-type giants are in excellent agreement with modern MK classifications. For the M dwarfs a new scale of spectral types, consistent with the giant scale, was established by means of the eight-color photometry (Wing 1973) and subsequently incorporated into the MK system by Boeshaar (1976). For M-type supergiants, the eight-color photometry has proved particularly useful: since the CN-based luminosity class can be calibrated in terms of absolute magnitude, while the reddening (and hence absorption) can be inferred from comparison of color and spectral type, the distance can be calculated from the photometric data alone. MacConnell et al. (1992) are taking advantage of this possibility to estimate distances to more than 1000 newly-discovered M-type supergiants in the southern Milky Way, including some which evidently lie in distant, highly obscured, spiral arms.

In the special case of stars that can be assumed to be unreddened, the eight-color photometry permits a different, and rather interesting, kind of two-dimensional classification. For M giants near the South Galactic Pole, the writer showed that the eight-color measures of $\mathrm{TiO}, \mathrm{CN}$, and continuum color can be used to classify the stars according to metallicity and $\mathrm{O} / \mathrm{C}$ ratio (Wing 1989a,b). The actual calibration of photometric indices in terms of metallicity and $\mathrm{O} / \mathrm{C}$ has not been attempted, however, but could be done with the help of synthetic spectra.

Eight-color observations of approximately 350 carbon stars were used 
TABLE 1. Data Available on Eight-Color System

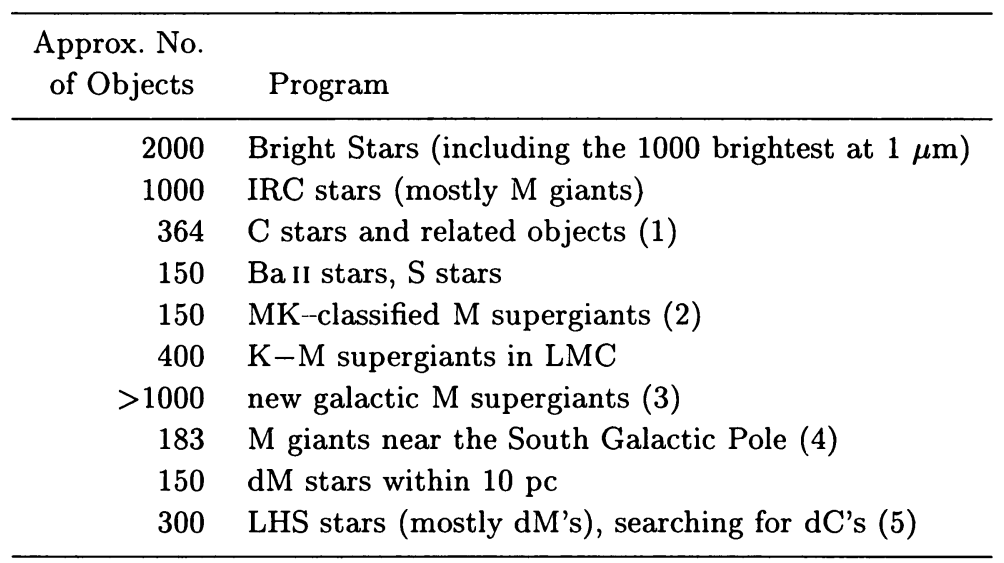

References: (1) Baumert (1972); (2) White \& Wing (1978);

(3) MacConnell et al. (1992); (4) Wing (1989a);

(5) Wing \& MacConnell (2000)

by Baumert $(1972,1974)$ to provide color temperatures and near-infrared magnitudes for a new determination of their absolute magnitudes from statistical parallax. Other applications of this large data set have not been exploited, however. Comparisons with synthetic spectra based on modern model atmospheres should allow determinations of effective temperatures and molecular abundances.

Stars of type S are awkward to study by narrow-band photometry because of the large number of molecules affecting the near-infrared spectrum. In fact, I had decided not to consider the $\mathrm{S}$ stars at all when choosing filters for the eight-color system. Consequently, the system in its normal form has no explicit measure of either $\mathrm{ZrO}$ or $\mathrm{LaO}$, and several of the filters are affected by bands which were long unidentified but are now known to be due to ZrS (Hinkle, Lambert \& Wing 1989). Piccirillo (1977), however, decided to "take the bull by the horns:" noting that two of the filters of the normal eight-color system (filters 4 and 6) are not particularly useful for S stars, he replaced them with new filters designed to measure $\mathrm{ZrO}$ at $6510 \AA$ and $\mathrm{LaO}$ at $7945 \AA$. In favorable cases, this modified eight-color system produces an infrared magnitude, a continuum color, and column densities for 5 molecules: $\mathrm{TiO}, \mathrm{ZrO}, \mathrm{LaO}, \mathrm{CN}$, and $\mathrm{ZrS}$ (Piccirillo 1980).

Table 1 is a summary of the observing programs that have been carried out with the (normal) eight-color photometric system. It does not include studies of individual objects or members of open or globular clusters, but only the programs which involved fairly extensive observing lists. In most cases the numbers of objects are not actual counts but estimates intended 
to indicate the scope of the project. Most of these data are unpublished but can be made available for collaborative studies.

A noteworthy characteristic is this observational effort is that nearly all of it has been done with small telescopes. During the first decade of this work, observations were made with an S-1 detector and were limited to rather bright stars. Although notoriously slow, good S-1 cells are extremely stable and, when used at good sites, produce excellent photometry. Early data were obtained primarily at Lowell Observatory and KPNO in the northern hemisphere and at CTIO in the southern; a considerable fraction of the observations of bright stars were made with telescopes of only 0.4-m aperture. Since about 1980, most of this work has been done at CTIO with the much more sensitive Varian LSE photocell. Recent programs, mostly using the $1.0-\mathrm{m}$ telescope during bright time, have included a study of the red supergiants in the LMC, a survey for galactic red supergiants, and a search for $\mathrm{dC}$ stars among Luyten proper-motion stars. With the widespread closure of small telescopes in recent years and the greatly decreased availability of aperture photometers, such data are becoming increasingly difficult to obtain, and existing data are becoming correspondingly more valuable.

\section{Continuum Measurements in the 1-4 $\mu \mathrm{m}$ region}

The 1-4 $\mu \mathrm{m}$ spectra of late-type stars contain absorption bands from numerous molecules, and several of these have been studied by means of narrowband photometry. Frogel and colleagues (Frogel et al. 1978; Aaronson et al. 1978; Cohen et al. 1978) showed that narrow-band indices of $\mathrm{CO}$ and $\mathrm{H}_{2} \mathrm{O}$ in the $2 \mu \mathrm{m}$ region, when combined with wide-band $J H K$ photometry, produce useful discriminants for population synthesis studies. Rinsland \& Wing (1982) used a spectrum scanner to make narrow-band measures of the first-overtone $\mathrm{SiO}$ bands near $4 \mu \mathrm{m}$, and Noguchi et al. $(1981,1986)$ have used spectrophotometry to measure the $3.1 \mu \mathrm{m}$ complex due to HCN and $\mathrm{C}_{2} \mathrm{H}_{2}$ in cool carbon and SC stars. On the other hand, the lines of some other important molecules such as $\mathrm{H}_{2}$ and $\mathrm{OH}$ are too scattered to be easily measured by this technique.

Measurements of the 1-4 $\mu \mathrm{m}$ continuum, when properly calibrated, are also of great interest. Since $\mathrm{H}^{-}$, the dominant opacity source throughout the 1-4 $\mu \mathrm{m}$ region in nearly all cool stars, has its opacity minimum at $1.65 \mu \mathrm{m}$, its effect is to distort the shape of the continuum, making it measurably different from a blackbody curve. Observationally, however, these distortions have proved difficult to measure quantitatively because the energy distributions need to be absolutely calibrated and molecular line absorption must be avoided. Wing \& Rinsland (1981) used a cooled grating spectrometer with an InSb detector at Kitt Peak to isolate 13 line-free 

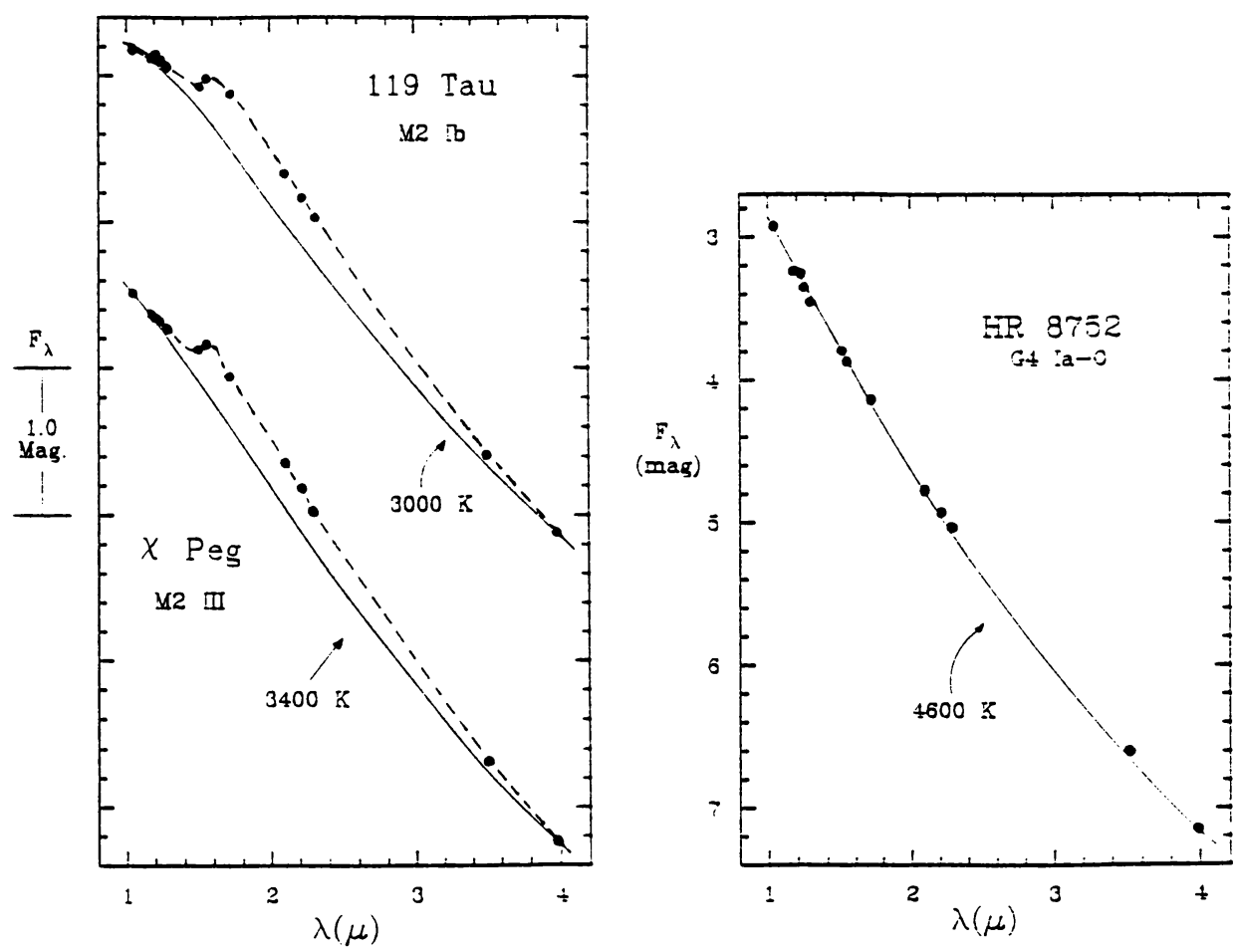

Figure 3. Energy distributions in the $1-4 \mu \mathrm{m}$ region, as measured with a spectrum scanner at 13 clean continuum points. Dashed curves are hand-drawn through the observed data; solid curves are blackbody curves fitted to the endpoints at 1.04 and $4.00 \mu \mathrm{m}$. The differences are due almost entirely to the effects of $\mathrm{H}^{-}$opacity. Note that HR 8752 shows no sign of $\mathrm{H}^{-}$and is evidently $\mathrm{H}$-poor.

continuum points between 1.04 and $4.00 \mu \mathrm{m}$ and found that their calibrated continuum spectra agreed well with model continua, clearly showing the effects of $\mathrm{H}^{-}$. Figure 3 shows three spectra obtained on this 13-color narrowband continuum program. The two stars in the left panel, a supergiant and a giant of type M2, both show clear deviations from a blackbody curve caused by $\mathrm{H}^{-}$. Similar deviations were found in several chemically-peculiar stars, including C stars, S stars, and Ba II stars. However, as pointed out by Wing \& Saizar (1989) and Wing (1991), some cool stars do not show any effect of $\mathrm{H}^{-}$, probably because of hydrogen deficiency. These include the HdC star HD 182040, the RV Tauri variables R Sct and U Mon, the RCB stars $\mathrm{R} \mathrm{CrB}$ and $\mathrm{XX}$ Cam, and the luminous supergiant HR 8752 (Fig. 3, right panel). Although HR 8752 has often been cited as one of the most luminous stars in the Galaxy, its infrared energy distribution suggests that it is not a normal supergiant. Rather, the blackbody shape of its 1-4 $\mu \mathrm{m}$ spectrum seems appropriate for the hydrogen-poor envelope of a post-AGB star. 


\section{Suggested Applications}

Narrow-band photometry can be used to compare real stars to model stellar atmospheres, and I have already alluded several times to the desirability of comparing the existing data to synthetic colors based on model atmospheres. Indeed, any quantitative interpretation of photometry must be based on modelling of some kind. At the same time, such comparisons can also be used to test the models themselves.

For many years, the state of models for cool stars with strong molecular bands was not sufficiently advanced to encourage detailed comparisons with observation. Simply stated, if the spectra computed from models don't look like real spectra, one does not feel inclined to use them to derive effective temperatures, abundances, etc. In recent years, however, the situation has changed dramatically. Improved methods for computing molecular opacities (e.g. Johnson 1986), including non-LTE effects (Johnson (1994) and greatly expanded molecular databases (Jørgensen 1995), have resulted in new grids of models for late-type stars (see, e.g. Gustafsson \& Jørgensen 1994; Plez 2000) which are believed to be good representations of the atmospheric structure and which generate realistic-looking synthetic spectra. It is then a simple matter to multiply the computed spectra by the response functions of any photometric system to produce "synthetic photometry" that can be compared directly to observation.

I believe that the data generated by the programs described here are ideally suited for comparisons with synthetic spectra, not only because they are internally precise and carefully calibrated to an absolute flux system but also because the filters (or scanner bandpasses) have been carefully chosen. Absorption bands are measured at their optimum positions, and each photometric system includes the best available continuum points.

When a good match is found between the synthetic colors of a model and the observed colors of an unreddened star, the effective temperature of the model can be considered the effective temperature of that star. Such determinations of $T_{\text {eff }}$ may not be as "fundamental" as determinations based on angular diameters, but they are likely to be more accurate. In most cases, effective temperatures differ significantly from the color temperatures provided directly by the photometry because of the effects of $\mathrm{H}^{-}$and other opacity sources affecting the continuum, and these $T_{\text {eff }}$ determinations are therefore needed to calibrate the color temperatures. Eight-color observations of bright standard stars have already been used to improve the $T_{\text {eff }}$ scale for K and M giants (Bell \& Gustafsson 1989), and suitable data are now available for similar determinations of the $T_{\text {eff }}$ scales for $M$-type dwarfs and supergiants, as well as individual values for hundreds of $\mathrm{C}$ stars and other chemically-peculiar late-type stars. Fitting the molecular band mea- 
surements with synthetic colors will convert them into column densities, leading to elemental abundances and possibly gravity indicators. We may even learn how a C-type Mira (Fig. 1) can manage to have the same color temperature at maximum and minimum light!

\section{References}

Aaronson, M., Frogel, J. A. \& Persson, S. E. 1978, ApJ, 220, 442

Baumert, J. H. 1972, Ph.D. dissertation, Ohio State University

Baumert, J. H. 1974, ApJ, 190, 85

Bell, R. A. \& Gustafsson, B. 1989, MNRAS, 236, 653

Boeshaar, P. C. 1976, Ph.D. dissertation, Ohio State University

Cohen, J. G., Frogel, J. A. \& Persson, S. E. 1978, ApJ, 222, 165

Frogel, J. A., Persson, S. E., Aaronson, M. \& Matthews, K. 1978, ApJ, 220, 75

Gustafsson, B. \& Jørgensen, U. G. 1994, Astron. Astrophys. Rev., 6, 19

Hinkle, K. H., Lambert, D. L. \& Wing, R. F. 1989, MNRAS, 238, 1365

Johnson, H. R. 1986, in The M-Type Stars, ed. H. R. Johnson and F. R. Querci, NASA SP-492, p. 323

Johnson, H.R. 1994, in IAU Coll. 146: Molecules in the Stellar Environment, ed. U. G. Jørgensen (Springer-Verlag), p. 234

Jørgensen, U. G. 1995, in Astrophysical Applications of Powerful New Databases, ed. S. J. Adelman and W. L. Wiese, ASP Conf. Ser., 78, 179

MacConnell, D. J., Wing, R. F. \& Costa, E. 1992, AJ, 104, 821

Noguchi, K. \& Akiba, M. 1986, Publ. Astron. Soc. Japan, 38, 811

Noguchi, K., Kawara, K., Kobayashi, Y., Okuda, H., Sato, S. \& Oishi, M. 1981, Publ. Astron. Soc. Japan, 33, 373

Piccirillo, J. 1977, Ph.D. dissertation, Indiana University

Piccirillo, J. 1980, MNRAS, 190, 441

Plez, B. 2000, in IAU Symp. 177: The Carbon Star Phenomenon, ed. R. F. Wing (Kluwer), p. 71

Rinsland, C. P. \& Wing, R.F. 1982, $A p J, 262,201$

Spinrad, H. \& Wing, R. F. 1969, Ann. Rev. Astron. Astrophys., 7, 249

White, N. M. \& Wing, R. F. 1978, ApJ, 222, 209

Wing, R. F. 1967, Ph.D. dissertation, Univ. of California, Berkeley

Wing, R.F. 1971, in Proc. Conf. on Late Type Stars, ed. G. W. Lockwood and H. M. Dyck (KPNO Contr. No. 554), p. 145

Wing, R. F. 1973, in IAU Symp. 50: Spectral Classification and Multicolour Photometry, ed. Ch. Fehrenbach and B. E. Westerlund (Reidel), p. 209

Wing, R.F. 1989a, in The Gravitational Force Perpendicular to the Galactic Plane, ed. A. G. D. Philip and P. K. Lu (L. Davis Press), p. 167

Wing, R.F. 1989b, Newsletter of Chemically Peculiar Red Giant Stars, No. 6, p. 7

Wing, R. F. 1991, in The Infrared Spectral Region of Stars, ed. C. Jaschek and Y. Andrillat (Cambridge), p. 301

Wing, R. F. \& MacConnell, D. J. 2000, in IAU Symp. 177: The Carbon Star Phenomenon, ed. R. F. Wing (Kluwer), p. 589

Wing, R. F. \& Rinsland, C. P. 1981, Rev. Mexicana Astron. Astrof., 6, 145

Wing, R. F. \& Saizar, P. 1989, in IAU Coll. 106: Evolution of Peculiar Red Giant Stars, ed. H. R. Johnson and B. Zuckerman (Cambridge), p. 151

Wing, R.F. \& Yorka, S. B. 1979, in IAU Coll. 47: Spectral Classification of the Future, ed. M. F. McCarthy, A. G. D. Philip and G. V. Coyne (Vatican Observatory), p. 519

Wing, R. F. \& Yuan, Y. 1998, in Pulsating Stars: Recent Developments in Theory and Observation, ed. M. Takeuti and D. D. Sasselov (Universal Academy Press), p. 113 


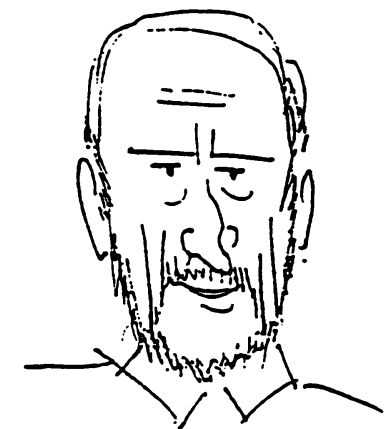

Tom LLOYD EVANS

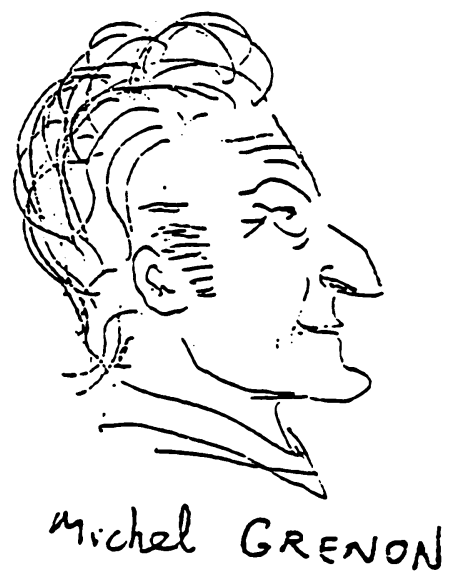

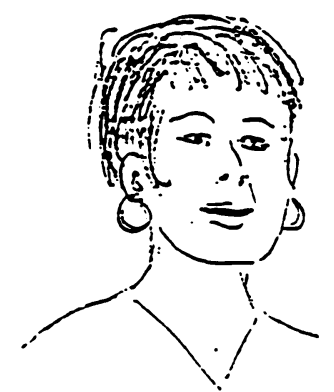

Isabelle CHERCHNEFF

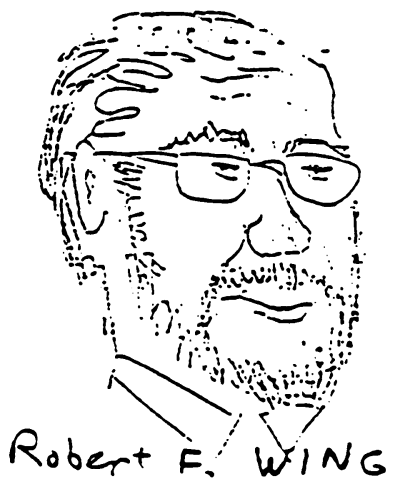

Four participants. Visual impressions by Pierre North. 